\title{
2-S13-2
}

\section{Practice and challenges of on-demand lectures (video lectures)}

\section{Satoshi Tanaka}

Dept. Pharmacol., Kyoto Pharmaceut. Univ.

The pandemic of Covid-19 has drastically changed the education program in universities. An on-demand lecture is one of the common approaches, which are employed to prevent the infection form spreading in the classroom. Ondemand lectures generally have a good reception of the students for the reason that they could repeatedly play back the video when needed and attend the class in a relaxed attitude as they like. On the other hand, we often have some concerns about their understanding of the lecture because we could not get the feedback during the preparation of the video lectures. In addition, majority of us, beginners of on-demand lectures, have to overcome the difficulties in utilizing various tools to support them.

Here, I would like to introduce some tips of on-demand lectures. One, who delivers a chalk talk, should obtain a good pen tablet and a suitable video recording software. Because video lectures with a large data size interrupt the comfortable views of the students, the video files should be compressed using a special software. Approachable Q\&A systems should greatly enhance the learning efficiency and open the door to the flip teaching. The on-demand lecture should remain a workable alternative after the pandemic ending. 